Rachel Goodman

University of Illinois, Chicago

\title{
Trading on Identity and Singular Thought
}

(Forthcoming in The Australasian Journal of Philosophy)

\begin{abstract}
On the traditional relationalist conception of singular thought, a thought has singular content when it is based on an 'information relation' to its object. Recent work rejects relationalism and suggests singular thoughts are distinguished from descriptive thoughts by their inferential role: only thoughts with singular content can be employed in 'direct' inferences, or inferences that 'trade on identity'. Firstly this view is insufficiently clear, because it conflates two distinct ideas-one about a kind of inference, the other a kind of process that grounds inferences - under the title, 'trading on identity'. Secondly, this leaves us without a notion that can be used as an alternative to relationalism about singular thought. The first notion is no more applicable to singular than to descriptive thought. The second may help us better understand singular thought, but does so, not by replacing the view that singular thoughts are information-based, but by helping us understand the nature of informationbased thought.
\end{abstract}

\section{Introduction}

Russell [1905, 1910, 1912] introduced a distinction between thoughts with singular and descriptive content. It came with an accompanying distinction between 'knowledge by acquaintance' and 'knowledge by description'. The idea was that having a thought with singular content was something that happened in virtue of bearing a special, epistemic-access relation to a thing. Without this relation, one could think of a thing only 'by description'. Thus, Russell's epistemic distinction explained or accounted for his semantic distinction: it told us what having a singular thought amounted to. The Kripkean philosophical revolution of the 1970s-80s made Russell's semantic distinction between thoughts with singular and descriptive content orthodoxy, but came with a challenge to his conception of it. In the wake of Kripke [1980] and others, it seemed to many that we could think non-descriptive thoughts about ordinary objects - and ones with which we lack special, intimate, epistemic-access relation at that.

Among those who think there is a legitimate category of singular thoughts, it is (more or less) agreed that the distinction between singular and descriptive thought should come with some account of what - epistemically, causally, cognitively, etc.—unifies and distinguishes singular thoughts. Beyond this, the debate about what having a singular, as opposed to descriptive, thought amounts to remains unresolved.

Recent approaches fall into two broad camps. The first weakens Russell's acquaintance requirement into the claim that singular thoughts are, by nature, information-based thoughts: they are about their objects in virtue of some causal-informational relation (like perception, testimony, etc.) between their thinker and what they're about. Inspired by Bach's [1987] distinction between relational and satisfactional aboutness, we'll call these theorists relationalists about singular thought. The second camp rejects the claim that all singular thoughts are based on causal-informational relations to their 
objects. Inspired in part by Kripke, they claim, for example, that thoughts expressed by descriptive names (names introduced by acts of descriptive reference-fixing) can be semantically singular. However, they aim to preserve the view that singular thought is a theoretically interesting, unified kind. They claim that what grounds or enables singular mental content is not a special causalinformational relation to an object, but rather some distinguishing cognitive, psychological or epistemic feature of a thought.

In this context, an influential but under-explained proposal has emerged in some of the most important recent work. This is that singular thoughts are distinctive in virtue of their inferential profile or cognitive role. In particular, singular thoughts are distinguished by the kind of inference in which they can feature: those that are 'direct' in the sense that they presuppose identity of reference across their premises, rather than state it. Following Campbell [1987], these are known as inferences that 'trade on identity'.

This proposal features in otherwise diverse work. It's at the heart of the recently dominant mental files conception of singular thought, but it is made most explicit in Dickie's [2015] epistemic account of singular thought. Despite its increasing influence, the proposal is insufficiently explained and defended. My aim is to outline, motivate and clarify it in enough detail to assess it. Firstly, I'll illustrate that proponents have not been clear enough about what trading on identity is and so not clear enough about what their proposal about singular thought is. Once we try to clarify 'trading on identity', we see that properly distinct ideas (one about a kind of inference, the other about a kind of process that grounds inferences) have been conflated under the title. Once we disentangle these, we are left without any notion that can be used to provide an alternative to relationalism about singular thought.

My conclusion is that 'trading on identity' cannot be exploited by opponents of relationalism to provide an alternative account of singular thought, but there are positive lessons to be learned. First, disambiguating the notion of trading on identity brings into focus questions about the processes_-reliable information sorting and management processes-required to maintain concepts that can feature in successful inferences. Second, an account of these processes may provide resources for a better understanding of singular thought_ - just not in the way opponents of relationalism assume. Perhaps we can better understand what's distinctive about singular thoughts by looking at the nature of the processes required to keep them in good order, but this will be part of an account of informationbased thoughts, not an alternative to the claim that singular thoughts are information-based.

\section{Trading on identity, First Pass...}

Singular thoughts, we're assuming, are thoughts (mental states) with singular content. Relationalists claim that what accounts for the singular content of a thought is that it is about the object it is singular with respect to in virtue of some causal-informational relation to it. What determines the reference of my singular thought about Obama is that I stand in a relation (like perception) to Obama allowing me to gain information from him. ${ }^{1}$ Opponents of relationalism reject this but, for the most part, recognize the burden of providing a replacement conception of what distinguishes singular thoughts — that is, of specifying some cognitive, epistemic or psychological 'singularity-making' feature that singular

1 There's disagreement about which particular relations count (e.g., perception, testimony, etc.). See Evans [1982], Bach [1987], Sawyer [2012]. 
thoughts share, and descriptive thoughts lack. An influential but under-explained proposal is that singular thoughts are inferentially distinctive: they alone can feature in a distinctively 'direct' form of inference known as an inference that 'trades on identity'.

To get this proposal on the table, I'll need to introduce the notion of 'trading on identity' in a preliminary way. As we proceed, we'll see this notion requires disambiguation.

Campbell [1987] and followers spell out trading on identity in terms of a contrast between cases in which the coreference of two token terms (linguistic or mental) can be legitimately assumed ('traded on') and cases in which it must be stated. ${ }^{2}$ The contrast is said to arise for arguments and inferences.

\section{Argument 1:}

$a$ is $\mathrm{F}$

$a$ is $G$

There is something that is $\mathrm{F}$ and $\mathrm{G}$

\section{Argument 2:}

$a$ is $\mathrm{F}$

$\underline{b \text { is } G}$

There is something that is $\mathrm{F}$ and $\mathrm{G}$

Argument 1 is formally valid whereas Argument-2 is not (even assuming 'a' and 'b' are coreferential). To make Argument 2 valid, we could add an 'identity premise', stating that $a=b$. This brings out that the formal validity of Argument 1 depends on an assumption of coreference being somehow encoded. ${ }^{3}$ An argument that encodes this assumption and thereby achieves formal validity 'trades on identity'.

Related to trading on identity is the notion of de jure coreference or direct coordination. This is the relation between the token terms whose coreference is 'traded on' in an argument that trades on identity: the relation that makes it the case no identity premise is required for the argument to achieve formal validity. Direct coordination relations can be present in arguments that do not trade on identity, however. An argument from the premises 'if $\mathrm{a}$ is $\mathrm{F}$, then a is G', and ' $\mathrm{a}$ is G', to the conclusion, ' $a$ is F', has directly coordinated terms across its premises but, on my understanding, is not one that trades on identity, because it doesn't use direct coordination relations to achieve formal validity. ${ }^{5}$ Direct coordination is a semantic relation holding between (token) terms. It can be present across token terms outside of the context of an argument, in the context of an invalid argument, or when the validity of an argument doesn't depend on it.

\footnotetext{
2 Campbell 1987: 276; Recanati 2012: 48; Dickie 2015: 81.

${ }^{3}$ In a formal language, it's encoded by the way the system works: the rules say sameness of syntactic type entails sameness of semantic type. For Campbell, it's encoded by sameness of sense.

${ }^{4}$ Note: if we add a premise to Argument 2 stating that $\mathrm{a}=\mathrm{b}$, the argument will trade on the identity of the referents of tokens of ' $\mathrm{a}$ ' and ' $\mathrm{b}$ '. This illustrates that trading on identity is a presupposition of formally valid arguments involving repetition of singular terms across premises.

${ }^{5}$ I'm not sure this is what Campbell and others would say (they don't discuss the case) but it helps make sense of positing both notions.
} 
In addition to arguments, 'trading on identity' is applied to minds and what they do (indeed, our interest is in the mental case). ${ }^{6}$ What is 'trading on identity' in the mental domain? Campbell talks about it as a feature of that which is frequently modeled by arguments: inferences (which we can think of, admittedly somewhat vaguely, as mental transitions that generate contentful mental states and transmit warrant, when they go well).

In the mental domain, we can understand trading on identity in terms of the contrast between making an inference appropriately modeled by Argument 1 and Argument 2. An inference modeled by Argument 1 trades on identity; one modeled by Argument 2 does not. Let's assume the equivalent of formal validity for inferences is manifest truth preservation from the subject's point of view, and that the inferential equivalent of a premise is a representation present in the antecedent state of the mental transition, which plays a role in that transition. Thus, an inference that trades on identity is one that has coreferential token terms in the representations playing the role of premises, and achieves manifest truth preservation without the need for a representation of the identity of the referents of those terms, partly by exploiting direct coordination relations between them.

Imagine I believe that a particular object I'm perceiving, say a snooker ball, is red and I also believe that the same ball is round. In some cases where I have two such beliefs, I'm in a position to infer, by way of a manifestly truth preserving inference of the following form, that there is something that is both red and round:

\section{Inference 1}

This is $\mathrm{F}$

This is G

Something is $F$ and $G$

If this is possible, my inference depends on my two beliefs being related in a way that somehow encodes the presumption that they refer to the same object. This relation between beliefs (or other mental states) is direct coordination for mental states. Inferences that trade on identity achieve manifest truth preservation from the subject's point of view partly by exploiting this relation. For a thinker to trade on identity is for her to make this kind of inference.

\section{Trading on Identity and Singular Thought}

An influential (but frequently inchoate) proposal is that singular thoughts are distinguished from descriptive thoughts by being the kind one can use to trade on identity.

The person to most clearly defend our proposal is Dickie [2015], who claims it is distinctive of singular thoughts that they can feature in inferences that trade on identity [Dickie 2015: 231-232, 243245]. On her view, justified inferences of this kind can occur only when the beliefs involved stand in underlying unity relations of the right kind: when they are directly coordinated. This happens only when the formation of those beliefs involves a 'justified information-marshaling strategy', whose role is to

\footnotetext{
${ }^{6}$ Campbell [1987: 279-80] talks of thinkers trading on identity.

7 Dickie uses 'direct coordination' to refer to the activity of bundling information to produce direct coordination relations, and 'unity relations' for direct coordination relations.
} 
form a body of beliefs that is treated by the subject (for inferential purposes) as coreferential [Ibid: 238]. Singular beliefs are the ones for which these kinds of information-marshaling strategies, yielding bodies of directly coordinated beliefs, exist. ${ }^{8}$ In contrast, 'merely descriptive beliefs' (those on the descriptive side of the singular/descriptive thought distinction) are not formed in a way that bundles them so as to support inferences that trade on identity.

Furthermore, Dickie is an opponent of relationalism about singular thought. ${ }^{10}$ She rejects the claim that singular thoughts are always about their objects in virtue of a causal, informational relation to them. She claims there are 'descriptively mediated singular thoughts'-sometimes expressed with descriptive names - for which this kind of relation is absent. These thoughts have singular content and are unified with other cases of singular thought (perceptual demonstrative and proper name-based cases) by the fact that they are bundled into directly coordinated 'bodies of beliefs' in virtue of being formed through the use of justified information-marshaling strategies.

A second instance of our proposal is Taylor's [2010] claim about the formal features of singular thought. Taylor claims that thoughts with singular form-thoughts that are 'fit' to non-descriptively refer to particular objects, whether or not they actually do-are 'devices of explicit coreference', capable of standing in 'chains of explicit coreference' [Taylor 2010: 80]. 'Explicit coreference' is Taylor's terminology for direct coordination [Ibid: 82]. It is a distinctive requirement on singular thoughts that they can be members of networks that stand in the relation of direct coordination-the relation that supports inferences that trade on identity [ibid: 83-4].

Finally, our proposal is at the heart of the increasingly popular 'mental files conception of singular thought' (MFC), which holds that all and only singular thoughts are file-based thoughts [Goodman 2016]. On the now-standard version of file theory (due largely to Recanati [2012, 2015]), the central theoretical role for mental files is to explain_or at least be the cognitive reality of - de jure coreference and trading on identity in thought. For file theorists, a thought that $a$ is $F$ and a thought that $a$ is $G$ are directly coordinated, and their thinker can trade on the identity of their referents, when the information 'is F' and 'is G' is co-filed. ${ }^{11}$ If what is distinctive of file-based thoughts is that they can stand in direct coordination relations, and what is distinctive of singular thoughts is that they are filebased thoughts, then what is distinctive of singular thoughts is that they can stand in direct coordination relations. ${ }^{12}$

I aim to clarify and evaluate a frequently made connection between singular thought and trading on identity, which is often gestured at rather than explained, and has therefore largely escaped critical examination. The authors above do us the service of making it explicit (to greater and lesser degrees).

But what, in more basic terms, motivates the connection between trading on identity and singularity? Why has it seemed to some that singular thoughts are distinguished by being those that can

\footnotetext{
${ }^{8}$ For Dickie, beliefs formed and bundled this way give rise to cognitive focus, which brings particular objects before the mind in a relation of robust 'aboutness' (what singular thought involves). See also [Dickie 2020].

${ }^{9}$ Dickie calls non-singular thoughts 'merely descriptive' because she claims some singular thoughts involve 'descriptionbased cognition'. This is her version of the claim that non-relational (in Bach's sense) thoughts can be singular.

10 This shouldn't be confused with the claim that singular thoughts can be 'satisfactional', which Dickie rejects.

11 Recanati 2012: 42-50.

12 The claim that only singular thoughts can be used to trade on identity is not always explicitly discussed by MFC proponents, but comes out in remarks like Jeshion's [2010:129] claim that descriptive, unlike singular, thoughts occur '... discretely in cognition...'.
} 
be used to trade on identity? Why think this provides an alternative to relationalism about singular thought?

The animating idea, I think, is an analogy between mental states that can feature in inferences that trade on identity, and sentences containing an individual constant in a logical language.

Start with the idea that we can use the language of formal logic to model mental states and processes (like inferences). In such a model, sentences containing individual constants model thoughts that are singular. Sentences containing individual constants can feature in arguments that trade on identity. Argument 1 involves repetition of the same individual constant across its premises and this is a way of encoding sameness of reference, so that there is no need to state it. An argument like Argument 1 is thought to appropriately model an inference like Inference 1, which trades on identity. The idea behind the proposal connecting trading on identity with singularity is that thoughts that feature in inferences that trade on identity employ an object-concept that plays a role analogous to an individual constant in a logical language. This might seem to provide an alternative to relationalism because it involves a conception of singular thought based, not on a thought's mode of referencedetermination (causal-informational vs not), but on its cognitive or inferential role.

However, to see the most basic form of the challenge to this proposal, note two points. First, the fact that sentences containing individual constants can feature in arguments that trade on identity does not imply that only sentences containing individual constants can. Second, this point tends not to arise in discussions of trading on identity because the notion is introduced (in Campbell [1987]) in the context of a discussion already limited to the singular case: it is defined as applying to arguments whose premises contain singular terms. ${ }^{13}$ Campbell asks, what does it take for an argument containing singular referential terms to be valid? His answer involves a notion apparently defined such that the question of whether arguments involving descriptive terms can trade on identity does not arise. This is legitimate because Campbell's question is about validity. But, for our purposes, if it is simply stipulated that trading on identity applies only to cases of singular thought, then it's not clear what's added to our understanding of singular thoughts.

This gives us a 'ground rule' for examining our proposal. The proposal is meant to help us understand what is distinctive about singular thoughts - what non-semantic feature unifies the category. If the ability to feature in inferences that trade on identity is the unifying feature, we need some independent (of the notion of singular reference/thought) grip on what trading on identity is. The ground rule is therefore that proponents of our proposal cannot rely on a stipulation that their notion of trading on identity does not apply to cases in which the thoughts used are descriptive.

\section{First Argument}

With exposition, documentation and motivation in place, we're now in a position to assess the proposal that singular thoughts are those that can be used to trade on identity.

I begin with a simple argument, which results from reflecting on the notion of trading on identity as it is generally presented. It suggests there's every reason to think this notion applies equally to cases in which the mental states involved in an inference are descriptive.

13 Campbell's discussion is packaged as about the role of non-descriptive sense, or 'the notion of the sense of a singular term' [Campbell 1987: 275, 278]. See also Recanati 2016: 5-7; Dickie 2015: 80. 
As we've seen, 'trading on identity' is explained in terms of arguments in which coreference can be legitimately assumed, in contrast to arguments where it must be stated. The idea is that some arguments achieve formal validity without the need to state the identity of the referents of token terms in their premises: they encode a presumption of identity without stating it. An inference that trades on identity is one that achieves manifest truth-preservation from the subject's point of view without the need for a representation that states the identity of the objects that are the referents of the mental states that serve as premises.

To see what's odd about the suggestion that only singular thoughts can be used to trade on identity compare Argument 1 and Argument 3.

\section{Argument 1:}

$a$ is $\mathrm{F}$

$a$ is $G$

There is something that is $F$ and $G$

\section{Argument 3:}

The $\mathrm{H}$ is $\mathrm{F}$

The $\mathrm{H}$ is $\mathrm{G}$

There is something that is $\mathrm{F}$ and $\mathrm{G}$

One of these arguments involves repetition of the same individual constant across premises; the other involves repetition of the same definite description. Both are formally valid. Furthermore, the validity of both 'turns on' an identity fact, and neither states that fact in a premise. Both arguments encode (rather than state) an identity fact that they rely on. If repetition of the same term-type across premises is what allows for trading on identity, then it seems that repetition of the same definite description and repetition of the same individual constant are ways to achieve the same end.

To get a contrast between an argument that trades on identity and one that doesn't, we need to instead compare Arguments 1 and Argument 2:

\section{Argument 1:}

$a$ is $\mathrm{F}$

$a$ is $\mathrm{G}$

There is something that is $F$ and $G$

\section{Argument 2:}

$a$ is $\mathrm{F}$

$b$ is $G$

There is something that is $F$ and $G$ 
Assuming that $\mathrm{a}=\mathrm{b}$, Argument 2 is truth-preserving, but not formally valid. To achieve formal validity, it requires an additional premise stating the identity of $a$ and $b$. But the same contrast applies to Argument 3 and Argument 4 (assuming that the $\mathrm{I}=$ the $\mathrm{H}$ ):

\section{Argument 3:}

The $\mathrm{H}$ is $\mathrm{F}$

The $\mathrm{H}$ is $\mathrm{G}$

There is something that is $F$ and $G$

\section{Argument 4:}

The $\mathrm{H}$ is $\mathrm{F}$

The $\mathrm{I}$ is $\mathrm{G}$

There is something that is $F$ and $G$

Undoubtedly, proponents of our proposal have the contrast between Argument 1 and Argument 2 in mind but, given their presentation of trading on identity, it's unclear what entitlement they have to exclude Argument 3.

Our first argument against the proposal that trading on identity can happen only with singular thoughts is simply that this point carries over when we consider inferences rather than arguments. An inference modeled by Argument 3-whose 'premises' are mental states with descriptive contentappears to stand on the correct side of the contrast used to spell out trading on identity. There's no apparent, special connection between inferences that trade on identity and singular rather than descriptive thoughts. Another way to put this: trading on identity arises in any case of formal validity exploiting the repetition of tokens of the same syntactic type (either linguistic or mental). This is too ubiquitous a phenomenon to tell us what is distinctive about singular thoughts.

Proponents of our proposal may respond that my argument distorts their intended notion of 'trading on identity'. They might claim that Argument 3 does not encode a presumption of sameness of reference because it doesn't involve reference at all. But, here, we must remember the broader dialectic and our ground rule. If trading on identity is to help us understand what is distinctive about singular thought, we cannot rely on a definition that stipulates a presumption of singular reference. There is no interesting proposal to be found about singular thought if our grip on trading on identity involves stipulation that descriptive cases don't count.

This leaves us with a first notion of 'trading on identity' — call it TI-1—and a first conclusion:

(TI-1) An inference trades on identity when it is manifestly truth preserving from the subject's point of view partly in virtue of encoding (not stating) sameness of extension across its premises.

Our first conclusion: if this is what is meant by 'trading on identity', then the claim that it is distinctive of singular thoughts that in using them their thinkers can trade on identity, is false. Any manifestly 
truth-preserving inference whose conclusion is not a logical truth seems to trade on identity. This shows why the phenomenon has nothing in particular to do with singular thought.

\section{A second notion of 'trading on identity'?}

If TI-1 is in question, it's false that only singular thoughts can be used to trade on identity. Why has this escaped notice? Perhaps proponents of our proposal have a somewhat different notion of trading on identity in mind. This means the usual presentation conflates TI-1 and this other notion. Part of my contribution is to point this out. Nonetheless, we should try to understand this second notion, and see if it vindicates their proposal about singular thought.

An alternative notion of 'trading on identity' - we'll call it TI-2 - is one on which it denotes, not a special kind of inference, but a distinctive kind of process that serves as partial ground or source for manifestly truth-preserving inferences.

To clarify TI-2, let's return to Arguments 1 and 3 and ask what, for proponents of our proposal, the relevant difference is meant to be. Both arguments are formally valid partly in virtue of encoding a presumption of identity of extension across their premises, so TI-1 applies to both. But consider the difference between how you would show their validity with a derivation in a formal system. Argument 3 would get a derivation that looks something like Derivation 3.

\section{Derivation 3:}

$\begin{array}{cl}(\exists \mathrm{x})[\mathrm{Hx} \&((\forall \mathrm{y})(\mathrm{Hy} \rightarrow \mathrm{x}=\mathrm{y}) \& \mathrm{Fx})] & (\mathrm{P} 1) \\ (\exists \mathrm{x})[\mathrm{Hx} \&((\forall \mathrm{y})(\mathrm{Hy} \rightarrow \mathrm{x}=\mathrm{y}) \& \mathrm{Gx})] & (\mathrm{P} 2) \\ \mathrm{Ha} \&((\forall \mathrm{y})(\mathrm{Hy} \rightarrow \mathrm{a}=\mathrm{y}) \& \mathrm{Fa}) & (\text { A for } \exists \text {-elim }) \\ \mathrm{Hb} \&((\forall \mathrm{y})(\mathrm{Hy} \rightarrow \mathrm{b}=\mathrm{y}) \& \mathrm{~Gb}) & (\text { A for } \exists \text {-elim }) \\ \ldots & \\ \mathrm{Hb} \rightarrow \mathrm{b}=\mathrm{a} & (\forall \text {-elim }) \\ \mathrm{Hb} & (\& \text {-elim, from-second-Ass. }) \\ \mathrm{b}=\mathrm{a} & (\rightarrow \text {-elim }) \\ \ldots & \\ \mathrm{Fa} \& \mathrm{Ga} & \\ \ldots & \\ (\exists \mathrm{x})(\mathrm{Fx} \& \mathrm{Gx}) & (\mathrm{C})\end{array}$

According to Dickie [2015: 244], the relevant difference is that Derivation 3 involves steps that dispense with the quantificational material in the premises. These steps involve reasoning about property instantiation and uniqueness. Let's call this 'descriptive reasoning'. In contrast, Argument 1's derivation wouldn't involve descriptive reasoning: it simply involves existential quantification over the occurrences of the individual constant in the premises. If 'trading on identity' is understood as a distinctive status some arguments have-whereby formal validity can be proven without descriptive 
reasoning — then Argument 1 has it but Argument 3 doesn't. ${ }^{14}$ However, for TI- 2 to produce a version of our proposal about singular thoughts it must have application in the mental domain.

But what is the analog for inferences of the distinction between arguments whose validity is proven via different kinds of reasoning? It seems unlikely the distinction is between inferences that do and don't involve representational steps (conscious or otherwise) like those in Derivation 3. We can surely reason from the beliefs that the $\mathrm{H}$ is $\mathrm{F}$ and the $\mathrm{H}$ is $\mathrm{G}$ to the conclusion that there is something that is $F$ and $G$ without representational steps concerning arbitrary objects, applying universal elimination, conditional-elimination, identity-elimination. ${ }^{15}$ Instead, Dickie [2015] claims that inferences modeled by Argument 1 and 3 have different 'justificatory profiles' and that this has to do with where their justification comes from, which in turn has to do with the way the thoughts involved in them are formed. ${ }^{16}$ Singular thoughts are the ones formed in the relevant way and therefore able to feature in inferences whose justification has the relevant source.

Thus, our next question: What is the source of justification for a manifestly truth preserving inference (in addition to the laws of logic)? In what sense can it come in different forms?

Care is required here. In addition to the laws of logic, manifestly truth-preserving inferences are licensed by direct coordination relations between the mental states serving as premises. It's possible for a thinker to rationally make Inference 1 or 3 only when she finds herself, at the time of inference, in a position to rationally presume that the object she believes to be $F$ and the object she believes to be $G$ are one. Direct coordination relations between her beliefs put her in that position. As we saw in Section-4, direct coordination relations hold between the beliefs serving as premises in the inference modeled by Argument 3, as well as Argument 1.

However, we can legitimately ask about the ground (or metasemantics) for direct coordination relations: in virtue of what do they hold? The answer is that, in thinkers like us, direct coordination relations exist in virtue of processes which collect information-often information encountered at different times_ and encode it as information concerning a single object. ${ }^{17}$

To be convinced, think what would happen (for thinkers like us) if there were no process in place that collected information and encoded it as information concerning the same object. Imagine I perceive an object at T1 and judge it to be F, and then moments later at T2, I perceive the same object and judge it to be G. If there were no process in place that connected these encounters and encoded the information acquired in them as being from a single object, then, when I found myself at T2 with the belief 'that is G', I wouldn't be in a position to infer from this and my earlier-formed belief that there is a single thing that is $\mathrm{F}$ and G. Manifestly truth preserving inferences are partially licensed by direct coordination relations, but these are grounded by processes that keep track of a single thing and

\footnotetext{
14 This satisfies our ground rule: Argument 3 doesn't count because it's derivation lacks independently specifiable features.

15 Dickie agrees [Ibid: 244].

16 Ibid: 231-38, 245.

${ }_{17}$ Note the restriction to 'thinkers like us'. I won't claim here that a 'swampman'-style thinker (or one whose history and interaction with her environment were substantially different to ours) couldn't have directly coordinated thoughts.
} 
cognize information received from it as concerning a single object. In slogan form: TI-1 is grounded by tracking processes. ${ }^{18}$

But what of TI-2? We can now frame the question of what TI-2 amounts to in the mental realm as follows: Are there different kinds of process that give rise to direct coordination relations? Are there processes that make use of descriptive reasoning (analogous to the steps in Derivation 3) and processes that don't? If so, TI-2 in the mental realm might be defined like this:

(TI-2) An inference trades on identity when the direct coordination relations between mental states that serve as its premises are grounded by a non-descriptive tracking process

The common notion of 'trading on identity' conflates TI-1 with TI-2: Campbell and followers gloss trading on identity in terms of inferences that are valid partly in virtue of encoding (not stating) sameness of reference across premises, but they also claim that a special kind of non-descriptive process is required for this to be the case. This is a conflation of the synchronic with the diachronic: TI-1 is a matter of what inferences an agent is in a position to make at a time; TI-2 is a matter of what kind of process puts her in that position.

Though they don't distinguish TI-2 from TI-1, there is evidence some theorists have something like TI-2 in mind. Campbell [1987: 283] claims that a process by which information is integrated is what accounts for the possibility of trading on identity and requires that this process be 'nonconceptual' or 'sub-personal'. Information-integration processes that involve reflection by the subject about whether information concerns a single object rule out that one is trading on identity. Similarly, Dickie [2015: 234-7] holds that, for beliefs to feature in inferences that trade on identity, their attached coordination process must be 'inferentially unmediated'.

Thus, our final task in clarifying TI-2, is to ask whether there is any legitimate distinction to be made between descriptive and non-descriptive tracking processes, and what it might look like. I'll formulate a distinction, and illustrate with an example:

\section{Descriptive Update Process}

The procedure by which new information is associated with the concept appeals to a 'matching' relation with information that's already associated with the concept. ${ }^{19}$

\section{Singular Update Process}

The procedure by which new information is associated with the concept does not appeal to a 'matching' relation with information that's already associated with the concept.

We can illustrate this contrast with an example from the cognitive-scientific literature: that of visual tracking, and the sense in which it has been claimed to be distinctively non-descriptive. What matters

\footnotetext{
${ }^{18}$ A 'tracking process', in my sense, need not be perceptual, or even causal/informational. A fully inferential process that works by deciding that some property $\mathrm{F}$ applies to the object that is the unique $\varnothing$ because of a general belief that all $\varnothing$ 's are $\mathrm{F}$ is a tracking process.

19 'Matching' is used broadly to include relations mediated by principles pairing different descriptive information.
} 
is not the details of this case, but the way it illustrates our contrast between descriptive update processes (which work by matching descriptive information) and singular update processes (which do not employ descriptive matching).

Many in the singular thought literature are familiar with Pylyshyn's [1989, 2006, 2007] claim that visual object tracking works by way of FINSTs, or 'visual indices': non-descriptive mechanisms, assigned to objects in context and used to track them. But what's less commonly discussed is the central distinction that illustrates the sense in which FINST's are meant to be non-descriptive. ${ }^{20}$ This is an argument that visual tracking employs a process that doesn't appeal to the matching of descriptive information.

The argument starts with the fact of our success in so-called 'multiple object-tracking' (MOT) tasks, and asks how our visual systems achieve this. In MOT tasks (illustrated in Fig. 4), a field of identical visual objects (with a subset cued as targets) move in independent and unpredictable trajectories. ${ }^{21}$ Subjects can successfully identify up to 4-5 target objects after this motion phase ends [Pylyshyn 2006: 226].

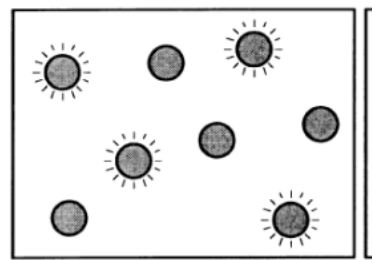

(a)

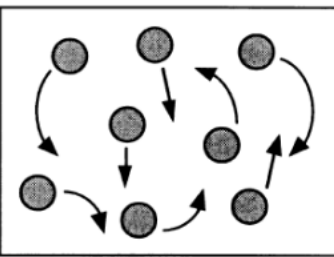

(b)

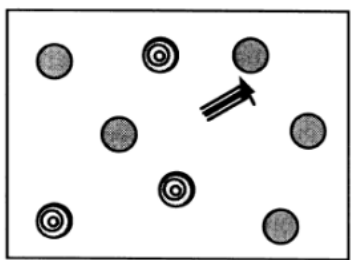

(c)

Fig. 4. A schematic depiction of a multiple-object tracking task. (a) Four items are initially flashed to indicate their status as targets. (b) All items then begin moving independently and unpredictably around the screen. (c) At the end of the motion phase, the subject must move the cursor about the screen to highlight the four targets - here the subject has just highlighted three of the targets, and is moving the mouse to the fourth. Animations of this task can be viewed or downloaded over the Internet at http:// pantheon.yale.edu/ bs265/bjs-demos.html.

Essentially, this means the visual system faces a correspondence problem that it must (and does) solve: that of computing correspondences between a series of synchronic visual representations. It must produce, for example, a correspondence between a visual representation of a circle at location $\mathrm{x}$ at $\mathrm{t} 1$, and one of a circle at location $y$ at $\mathrm{t} 2$, such that the later representation is taken to be of the same object. The question is, how? Pylyshyn outlines two possible kinds of solution, which illustrate the distinction between descriptive and singular update processes.

What I'll call a descriptive solution computes correspondences by a process whereby descriptive information is encoded, and used in a matching algorithm. The matching algorithm pairs a later representation with an earlier representation by comparing (matching) descriptive information encoded at different times. For example, if the system were dealing with differently colored and shaped objects, it could encode at $\mathrm{t} 1$ that there was a red circular object at location $x$ and, when it spotted a

${ }^{20}$ It first appears in Pylyshyn \& Storm [1988].

${ }^{21}$ Fig. 4 from Scholl 2001. 
red circular object at location $y$ at $\mathrm{t} 2$, it could conclude that the object at $\mathrm{t} 2$ is identical to the object at $\mathrm{t} 1$, because of the descriptive match. ${ }^{22}$

What I'll call a non-descriptive solution is largely defined negatively, as one that does not work by encoding property information and using it in a matching algorithm. The idea is that, in visual tracking, correspondences between synchronic representations are generated by a process that does not rely on encoding property information, and comparing that information [Pylyshyn 2006, 2007]. ${ }^{23}$

Pylyshyn and Storm [1988] offer an empirical argument that visual tracking involves a nondescriptive solution to the correspondence problem (the details of which need not concern us here). ${ }^{24}$ The point for our purposes is, 1) that a process solving the visual correspondence problem is an example of the kind of process that grounds direct coordination relations in the case of conceptual thought, and 2) that Pylyshyn formulates a sense in which such a process can itself be non-descriptive. Visual tracking processes, like tracking processes underwriting direct coordination relations, must reliably treat information from the same object as such, and information from different objects as such. If tokens of the same object-concept (type) are to stand in the direct coordination relations that underwrite manifestly truth-preserving inferences, concepts must be associated with processes (ie., with solutions to what is essentially a correspondence problem) that track objects and organise information such that it is encoded as concerning a single object. For new information that arises, this process - that is, the update process for the concept—-must reliably answer the question, 'should this information be associated with this concept?'. ${ }^{25}$ Pylyshyn's idea is that any update process that doesn't work by matching new information with descriptive information already associated with the concept is distinctively non-descriptive. We can take this as a model for TI-2 in the mental domain.

This in turn gives us a new version of our proposal about singular thought: singular thoughts are thoughts about particulars that employ a singular update process.

\section{Problems: Relationalism, and Categorising Cases}

Our second version of the claim that trading on identity is distinctive of singular thought involves understanding the singular/descriptive thought distinction in terms of the kind of update process that a thought employs. This 'diachronic' conception of singular thought is worthy of consideration but it isn't available to opponents of relationalism as an alternative conception of singular thought.

\footnotetext{
22 A descriptive matching algorithm could also produce correspondences between different descriptive information. E.g., a process employing an algorithm applying the principle of looking at a later time for the object at the nearest location and thereby matching descriptions specifying different locations would be descriptive. See Pylyshyn [2006: 224-5].

${ }^{23}$ You might object that property discrimination must have some role in object-tracking: how else could we account for the figure from ground discriminations presupposed? Pylyshyn's claim is that property discrimination plays a merely causal, rather than computational, role [Pylyshyn 2006: 218; 2007: 17, 33, 73]. It might be that the system's states are responsive to certain properties, but the tracking process doesn't rely on encoding and employing property information in computations producing correspondences. Whether or not this is true, it's coherent and doesn't imply that the mind somehow tracks heccaeties.

24 The crux is that human attention-scanning speeds would prevent the successful application of any descriptive procedure. Given the manner and speed of motion of objects in MOT experiments, a descriptive procedure would mix objects up, distractor objects ending up in locations producing the assumption they were targets. See also [Pylyshyn 2006: 223-4; 2007: 36-7]

${ }_{25}$ Dickie [2015] calls this an 'information-marshaling strategy'.
} 
To see why, think about the role of an update process, and the options for how it can successfully play that role. The role is to reliably sort information so that information from the same object is treated as such, and information from distinct objects is treated as such. Next, we can ask, for creatures like us, could there be a way of reliably adding information about a particular object to a body of information, which doesn't appeal to a 'matching' relation with information already associated with that body, and also doesn't rely on a causal, informational connection to the object the body is about? It seems not. If the explanation of why information is added to the body doesn't appeal to the fact that it is coming in through a certain informational connection, then it must surely appeal to a relation between the content of the information and the content of information already contained in the body - that is, to a descriptive 'match'. Otherwise, what would underwrite the reliability of the process? Why would the agent be justified in assuming a single object was at issue? Perhaps there's a possible creature who adds new information to her bodies of information neither in virtue of the descriptive relation of that information to information she already possesses, nor in virtue of the fact that the information comes in through the same causal informational connection, but this creature would be either magical or irrational.

In support of this, it's instructive that Pylyshyn relies on an analogy with touch to illustrate the very idea of a non-descriptive solution to the correspondence problem ('FINST' stands for finger of instantiation). FINSTs are said to allow you to keep track of an object without employing property information in something like the way you could track an object if you simply kept your finger on it. In this case, you would rely on a continuing connection with the object, and thereby override the need for a computation that compares and pairs information. The continuing connection plays the reliability-underwriting role otherwise played by descriptive-matching. My point is, similarly, that a reliable singular update process must rely on a causal, informational connection to an object to adjudicate whether some piece of information concerns the same object again. This means a TI-2based conception of singular thought is not an alternative to relationalism.

The idea of non-descriptive update processes might be part of a clearer account of what it amounts to for a thought to be 'information based', and thereby part of an account of singular thought, but this will not be an alternative to relationalism.

In any case, if update processes are used to distinguish singular thought, problems with the application of the distinction between singular and descriptive update must be addressed. Classifying thoughts as singular or descriptive according to the singular/descriptive update distinction requires us to endorse the seemingly false claim that each body of directly coordinated thoughts—each conceptis associated with a single update process.

An ordinary example illustrates. Imagine a perceptual case, where I form beliefs about an object on the basis of continued perception. I form the belief that the object is red, then the belief it is round. These beliefs are directly coordinated because underpinned by a process that encodes the information 'is red' and 'is round' as concerning the same object. On the assumption that perceptual demonstrative thoughts like these are singular, and that what makes for singularity is update process, the update process for these thoughts should be singular. On this view, when new information arises ('is round'), it is integrated (or not) simply because it does (or doesn't) come in through the same perceptual channel. 
The update process doesn't appeal to any 'matching' relation with information already associated with the concept.

But imagine I receive the information that every round object in the room is an apple. I form the belief that this object is an apple, on the basis of a match (via the principle of logical implication) between the information 'is an apple' and 'is round' (which is already associated with my object concept). My beliefs are directly coordinated: I can validly infer there's something that is a red apple. However, the process grounding this inference is mixed, not wholly singular or descriptive. This is an ordinary case, the structure likely ubiquitous, resisting classification as singular or descriptive.

In response, it could be claimed that cases should be classified by their dominant update process. ${ }^{26}$ For example, what it means for a cluster of coordinated thoughts to be perceptual demonstrative thoughts is that perceptual information has 'trumping status' with respect to other information. In our case, the update process might be 'mixed' but if, in addition to the inferentially sourced 'is an apple', I were to receive perceptual information to the effect that the object were a plum, the perceptual information would trump the inferential information. Thus, the dominant update strategy is singular.

However, the problem isn't so easily solved. The response requires that there is a stable, dominant update process for each cluster of directly coordinated mental states. But why think dominance relations are stable? For example, does perceptual update stably trump inferential update in cases of perceptual attention to an object? The descriptively produced 'is an apple' might trump the singularly produced 'is a plum' if I'm told by a trusted party that someone is manipulating the lighting. Across the course of a process generating direct coordination relations, dominance relations may change. One might learn that the lighting is misleading. Even at a time, there may be no straightforward facts about which update strategy trumps others. For Example, which information takes precedence might depend on the nature of the properties involved. Perhaps information from perception trumps information from testimony when it comes to conflicting color information, but the testimonial channel trumps the perceptual channel with respect to conflicts over species kinds (oak vs. elm). Thus, update processes themselves might not stand in dominance relations, even synchronically.

We can conclude that TI-2 does not provide a workable version of the claim that trading on identity is distinctive of singular thought, and certainly not one that is an alternative to relationalism. ${ }^{27}$

\section{Addressing a Final Response}

I have given arguments against both versions of the proposal that 'trading on identity' can be used to replace the relationalist view of singular thought. However, I want to trace out one final line of thought, which perhaps lies behind the claim that trading on identity occurs only in the singular case. This will bring out what, in a basic sense, is wrong with the claim.

One might be tempted to think that the update processes that form direct coordination relations are not required, or have no role to play, in the case of thoughts with descriptive content. Take two cases, and stipulate that the first involves two co-extensional singular beliefs ('this is F', 'this is G'), and the

\footnotetext{
${ }^{26}$ This is inspired by Evans's [1982] idea of a controlling conception. See also Dickie's [2015] idea of a proprietary means of justification.

27 There is space for a still-weaker classification whereby singular concepts are those that involve, or permit, some degree of singular update.
} 
other two co-extensional descriptive beliefs ('the $\mathrm{H}$ is F', 'the $\mathrm{H}$ is $\mathrm{G}$ '). Why think the descriptive beliefs aren't attached to update processes and don't stand in direct coordination relations? One might think coordination into a body of beliefs is required in the singular case, because there are no other resources available for securing an assumption of co-reference. If one hasn't kept track of an object, then when one finds oneself with a series of 'this is F', 'this is G', beliefs, one has no resources to determine whether these beliefs concern the same object. In the descriptive case, resources involving reasoning about property instantiation and uniqueness are available to answer this question. After all, if this object is the unique $\mathrm{H}$ and that object is the unique $\mathrm{H}$, then this object must be that object.

However, if a thinker has two token thoughts to the effect the $\mathrm{H}$ is $\mathrm{F}$ and the $\mathrm{H}$ is $\mathrm{G}$, her assumption of coreference is just as dependent on her having successfully kept track (and thereby on having directly coordinated token states) as in a case where she has two thoughts to the effect that $a$ is $\mathrm{F}$ and $a$ is G. If I have a belief whose referential content is that the only doctor in town is well trained, and a belief whose referential content is that the only doctor in town is honest, am I automatically (without tracking or direct coordination) in a position to make an inference to the conclusion that someone in town is honest and well trained? I am not, because Frege cases can occur for properties too. ${ }^{28}$ Suppose my situation is that I would express the first belief with 'the only doctor in town is well trained', and the second with 'the only physician in town is honest'. In such a case, I might not be in a position to make the inference, because I might have failed to keep track of the fact that I am thinking of the same property in both cases.

This comes to nothing more than the fact that a Frege case is just as possible when thinking about an object descriptively. That one's thoughts are descriptive doesn't do away with the need for update processes and direct coordination.

You may think I've overlooked the crucial difference between singular and descriptive cases: that the target of coordination is an object in the singular case, whereas it is a property in the descriptive case. This may be true, but does not vindicate the claim that only singular thoughts come in coordinated bodies and rely on update processes. That coordination in the singular case targets objects directly, whereas it targets objects via their properties in the descriptive case, is simply another way of saying that singular thoughts are directly about objects, whereas descriptive thoughts are really about properties. But this was our starting assumption: it is simply another way of saying that singular thoughts have singular contents, whereas descriptive thoughts have descriptive contents.

It is ultimately unsurprising that appeal to coordination of mental states into 'bodies' that stand in direct coordination relations does not itself distinguish singular from descriptive thought. Appeal to direct coordination and trading on identity is made in the service of answering Frege's Puzzle, which is ubiquitous, potentially arising not only in some limited domain, but in all our interactions with an external world on which our cognitive grip is fallible and perspectival.

\section{Conclusion}

In clarifying 'trading on identity', we've uncovered two distinct notions, usually conflated. TI-1, or the notion of an inference that is manifestly truth preserving partly in virtue of encoding (not stating) sameness of extension across its premises. And TI-2, or the notion of a non-descriptive 'update

28 See Mates 1952. 
process' used to collect information and encode it as concerning the same object. All inferences to which TI-1 applies presuppose some update process, but whether the update process is singular, descriptive, or both, is a separate question.

In disambiguating 'trading on identity', we've explored several possible claims about the nature of singular thought, also usually conflated.

A first is that singular thoughts are those that can feature in a certain kind of inference (characterized by TI-1). We've seen that TI-1 is too general a phenomenon for this to be true: it applies to any manifestly truth-preserving inference whose conclusion is not a logical truth, because Frege's puzzle is ubiquitous, not restricted to cases of singular thought.

A second is that singular thoughts are those featuring object-concepts that employ singular update processes. Uncovering the suggestion that singular update is distinctive of singular thought might pave the way for an account of what it means for a thought to be information-based, but this account must face the challenge of sorting cases: it cannot assume that each body of directly coordinated beliefs comes with a single update process (or a dominant one). More importantly, the proposal cannot be exploited by opponents of relationalism, and so doesn't vindicate existing versions of the claim that trading on identity distinguishes singular thought. Whatever truth there is in the suggestion that singular thoughts are better understood by looking at their diachronic dimension (and there may be some), it is not an alternative to conceiving of them as information-based thoughts.

\section{Acknowledgements:}

Thanks to Imogen Dickie, Aidan Gray, Robin Jeshion, Josef Stern, Mark Sainsbury, Ken Taylor, Malte Willer, and audiences at the University of Texas at Austin, the University of Wisconsin at Milwaukee and the University of Chicago, for discussion of the issues and questions. I also thank two anonymous referees for AJP for very helpful comments, and Stephen Hetherington for especially valuable editorial advice that helped me shorten and improve the paper.

Note: The paper was shortened for publication. A longer version is available at my personal website.

\section{References}

Bach, K. 1987. Thought and Reference, Oxford: Clarendon Press.

Campbell, J. 1987. Is Sense Transparent?, Proceedings of the Aristotelian Society 88: 273-92.

Dickie, I. 2015. Fixing Reference, Oxford University Press.

Dickie, I. 2020. Cognitive Focus, in Singular Thought and Mental Files. Eds. Rachel Goodman, James Genone and Nick Kroll, Oxford: Oxford University Press.

Evans, G. 1982. The V arieties of Reference, Ed. John McDowell, Oxford: Clarendon Press.

Goodman, R. 2016. Against the Mental Files Conception of Singular Thought, Review of Philosophy and Psychology 7/2: 437-61.

Hawthorne, J. and Manley, D. 2012. The Reference Book, Oxford: Oxford University Press.

Jeshion, R. 2004. Descriptive Descriptive Names, in Descriptions and Beyond. Ed. Marga Reimer and Anne Bezuidenhout, Oxford: Clarendon Press. 
Jeshion, R. 2010. Singular Thought: Acquaintance, Semantic Instrumentalism and Cognitivism, in New Essays on Singular Thought. Ed. Robin Jeshion. Oxford: Oxford University Press: 105-40.

Kripke, S. 1980. Naming and Necessity. Cambridge, MA: Harvard University Press.

Mates, B. 1952. Synonymity, in Semantics and the Philosophy of Language, Ed. Leonard Linsky. University of Illinois Press.

Pylyshyn, Z. W. and Storm, R.W. 1988. Tracking Multiple Independent Targets: Evidence for a Parallel Tracking Mechanism, Spatial Vision,3/3: 179-97.

Pylyshyn, Z. W. 1989. The Role of Location in Spatial Perception: Sketch of the FINST Spatial-Index Model, Cognition 32/1: 65-97.

Pylyshyn, Z. W. 2006. Seeing and Visualizing: It's Not What You Think, Bradford: MIT Press.

Pylyshyn, Z. W. 2007. Things and Places: How the Mind Connects with the World, MIT Press.

Recanati, F. 2012. Mental Files, Oxford: Oxford University Press

Recanati, F. 2015. Cognitive Dynamics: A New Look at an Old Problem, in Reference and Representation in Thought and Language. Eds. K. Korta \& M. Ponte. Oxford University Press.

Recanati, F. 2016. Mental Files in Flux. Oxford: Oxford University Press.

Russell, B. (1905) 'On Denoting', Mind. 14/56: 479-93

Russell, B. (1910) 'Knowledge by Acquaintance and Knowledge by Description', Proceedings of the Aristotelian Society. Vol. 11: 108-28

Russell, B (1912) The Problems of Philosophy. Oxford: Oxford University Press.

Sawyer, S. 2012. Cognitivism: A New Theory of Singular Thought?, Mind and Language 27/3: 264-83.

Scholl, B.J. 2001. Objects and Attention: The State of the Art, Cognition 80/1-2: 1-46.

Taylor, K. 2010. On Singularity, in New Essays on Singular Thought. Ed. Robin Jeshion. Oxford: Oxford University Press: 77-102 